\title{
HADES Overview
}

\section{Recent results from $\mathrm{Ag}+\mathrm{Ag}$ collisions at $\sqrt{\mathrm{S}_{\mathrm{NN}}}=2.55 \mathrm{GeV}$ measured by HADES}

\author{
Simon Spies $^{1, *}$ for the HADES collaboration \\ ${ }^{1}$ Institut für Kernphysik, Goethe-Universität, 60438 Frankfurt, Germany
}

\begin{abstract}
In March 2019 the HADES experiment recorded 14 billion Ag+Ag collisions at $\sqrt{\mathrm{S}_{\mathrm{NN}}}=2.55 \mathrm{GeV}$ as a part of the FAIR phase- 0 physics program. With the capabilities to measure and analyze particles forming the bulk matter, namely pions, protons and light nuclei, as well as rare probes like dilepton decays of vectormesons and strange hadrons, the HADES experiment allows to study the properties of matter at high densities in great detail. In this contribution a special focus is put on the reconstruction of weakly decaying strange hadrons.
\end{abstract}

\section{Introduction}

In heavy-ion collisions at bombarding energies of a few $\mathrm{GeV}$ as measured by HADES, matter is compressed to densities above its groundstate density. At the same time, it is heated to temperatures of $70 \mathrm{MeV}$ extracted using dilepton radiation [1]. Similar densities and temperatures are also expected in merging neutron stars [2,3]. Therefore, the study of heavy-ion collisions in the few $\mathrm{GeV}$ regime allows to deduce information on the microscopic composition and ultimately also macroscopic properties like for example the equation of state of astronomic objects like merging neutron stars.

\subsection{Strangeness Production}

In this energy regime, strange hadrons are produced close to or even below their free nucleon nucleon threshold energy which results in a steep rise of their excitation function with increasing $\sqrt{\mathrm{s}_{\mathrm{NN}}}$. Thus, even slight effects connected to the surrounding medium significantly influence their production [4].

In previous measurements of HADES a stronger than linear rise of the production rates of strange hadrons with the average amount of nucleons participating in the collision $\left\langle\mathrm{A}_{\text {Part }}\right\rangle$ [5] was observed and might be connected to these medium effects. Furthermore, the strong excess of baryons in the fireball leads to different potentials for strange and anti-strange mesons [6-8], which was studied by multiple experiments [9-14].

\footnotetext{
*e-mail: s.spies@gsi.de
} 


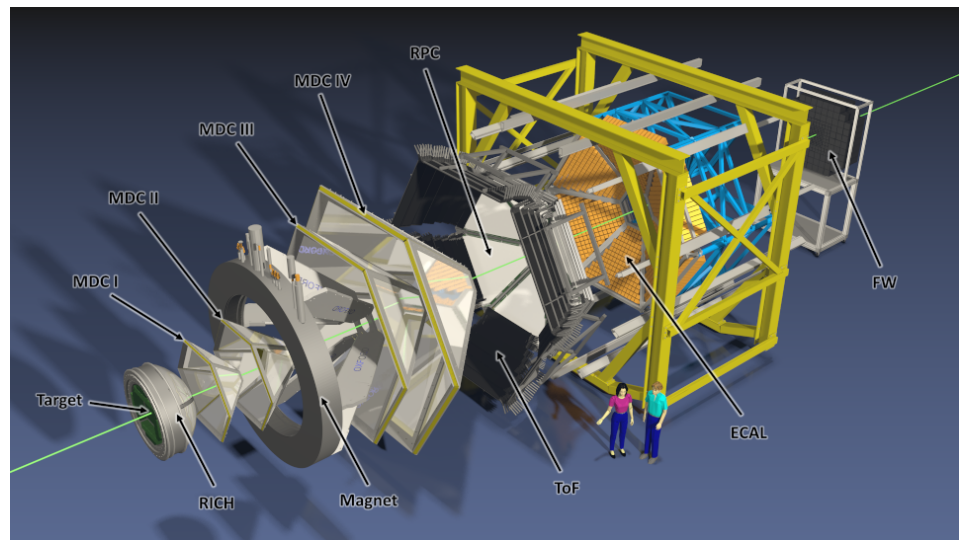

Figure 1. Schematic expanded view of the HADES detector setup.

\section{HADES Experiment}

The HADES experiment, which is schematically depicted in an expanded view in figure 1 , is a multi-purpose fixed-target magnet spectrometer operated with the SIS18 accelerator at GSI, Darmstadt, Germany. Besides heavy-ion A+A collisions, also elementary $\mathrm{p}+\mathrm{A}$ and $\mathrm{p}+\mathrm{p}$ as well as pion induced $\pi+A$ collisions are measured, e.g. [15-17]. Starting from the segmented 15-fold silver target, the particles traverse a Ring-Imaging CHerenkov (RICH) detector for electron/positron identification, two low-mass Mini Drift Chamber (MDC) tracking stations, a toroidal magnetic field, two further MDC tracing stations and depending on the polar angle either a Resistive Plate Chamber (RPC) or a Time of Flight (ToF) scintillation detector for time of flight measurement. The setup is completed by an Electromagnetic CALorimeter (ECAL) for photon detection, a Forward Wall (FW) hodoscope for measurement of the event plane and a diamond reaction time and trigger detector (START) located in front of the target. Besides the RICH, the FW and the START detector, all detectors are splitted into six independent sectors covering almost the entire azimuthal angle. More details on the HADES experiment are given in [18].

\section{Weak Decay Recognition}

In contrast to the charged kaons which have a mean flight length $c \tau$ of few meters and are thereby measured directly in the detectors, the $\Lambda$ hyperon and $K_{S}^{0}$ meson have a $c \tau$ of only few centimeters and thereby need to be reconstructed from their decay daughters. Due to the large amounts of protons and pions not associated with weak decays, a method to strongly separate the signals from the background is required. As a result of the rather high $c \tau$ values of weakly decaying particles, a spacial displacement between the primary event vertex and the secondary decay vertex can be observed. This displacement results in a so-called OffVertex-Decay-Topology which is quantified using the following parameters:

- Distance between the primary and secondary vertex (VDX).

- Distance of closest approach (DCA) between the daughter tracks and the primary vertex. (Dau1VD and Dau2VD)

- DCA between reconstructed mother track and primary vertex. (MotVD)

- DCA between the two daughter tracks. (MTD)

- Opening angle between the two daughter tracks. (A) 


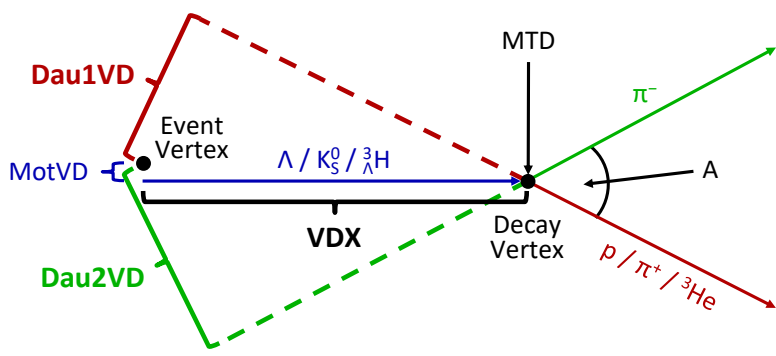

Figure 2. Schematic depiction of the Off-Vertex-Decay-Topology of $\Lambda, K_{S}^{0}$ and ${ }_{\Lambda}^{3} H$ decays.

In addition, the method proposed by R. Armenteros and J. Podolanski [19] is used to take the kinematic constraints of decays into account. In the ultrarelativistic limit, these constraints reduce to the ellipse equation 1 with the parameters defined by equation 2 .

$$
\begin{gathered}
\left(\frac{\alpha-\alpha_{0}}{r_{\alpha}}\right)^{2}+\frac{p_{\perp}^{2}}{p_{c m s}^{2}}=1 \\
p_{c m s}=p_{c m s}^{ \pm}=\sqrt{\left(\frac{M^{2}+m_{ \pm}^{2}-m_{\mp}^{2}}{2 M}\right)^{2}-m_{ \pm}^{2}} \text { and } \alpha_{0}=\frac{m_{+}^{2}-m_{-}^{2}}{M^{2}} \text { and } r_{\alpha}=\frac{2 p_{c m s}}{M}
\end{gathered}
$$

Since the ultrarelativistic limit is not reached at typical HADES collision energies, we adapt the original formalism by introducing an artificial boost of $\beta=0.99$ in longitudinal direction on the measured Lorentz vectors before calculating the Armenteros-Podolanski variables. Next, the characteristic Armenteros-Podolanski ellipses are interpreted in polar coordinates by assigning them a radial and an azimuthal component in the $\alpha$ - $p_{\perp}$-plane according to equation 3 .

$$
r_{\alpha p_{\perp}}=\sqrt{\left(\frac{\alpha-\alpha_{0}}{r_{\alpha}}\right)^{2}+\frac{p_{\perp}^{2}}{p_{c m s}^{2}}} \text { and } \phi_{\alpha p_{\perp}}=\arctan \left(\frac{p_{\perp}}{p_{c m s}} \cdot \frac{r_{\alpha}}{\alpha_{0}-\alpha}\right)
$$

The radial component $r_{\alpha p_{\perp}}$ is strongly correlated to the invariant mass of the daughter particles and thereby inappropriate to separate signal and background. However, the azimuthal component $\phi_{\alpha p_{\perp}}$, in the following APAngle, is at most weakly correlated to the invariant mass and thereby well suited as a further parameter to reject background. To our knowledge, such a modification has not been discussed in literature yet.

Finally, the rejection of background is enhanced further by using a Multi-LayerPerceptron (MLP) artificial Neural Network (aNN) from the Toolkit for Multivariate Data Analysis (TMVA) [20]. Therefore, the Off-Vertex-Decay-Topology parameters Dau1VD, Dau2VD, MotVD, VDX and MTD as well as the azimuthal angle of the ArmenterosPodolanski ellipse APAngle are used as input parameters and the aNN is trained using simulated signal samples and mixed-event background samples.

In previous analyses $[5,21,22]$ it was shown that the use of an aNN to reject background in the reconstruction of weak decays significantly improves the reconstructed signals compared to pure hard-cut analyses. 

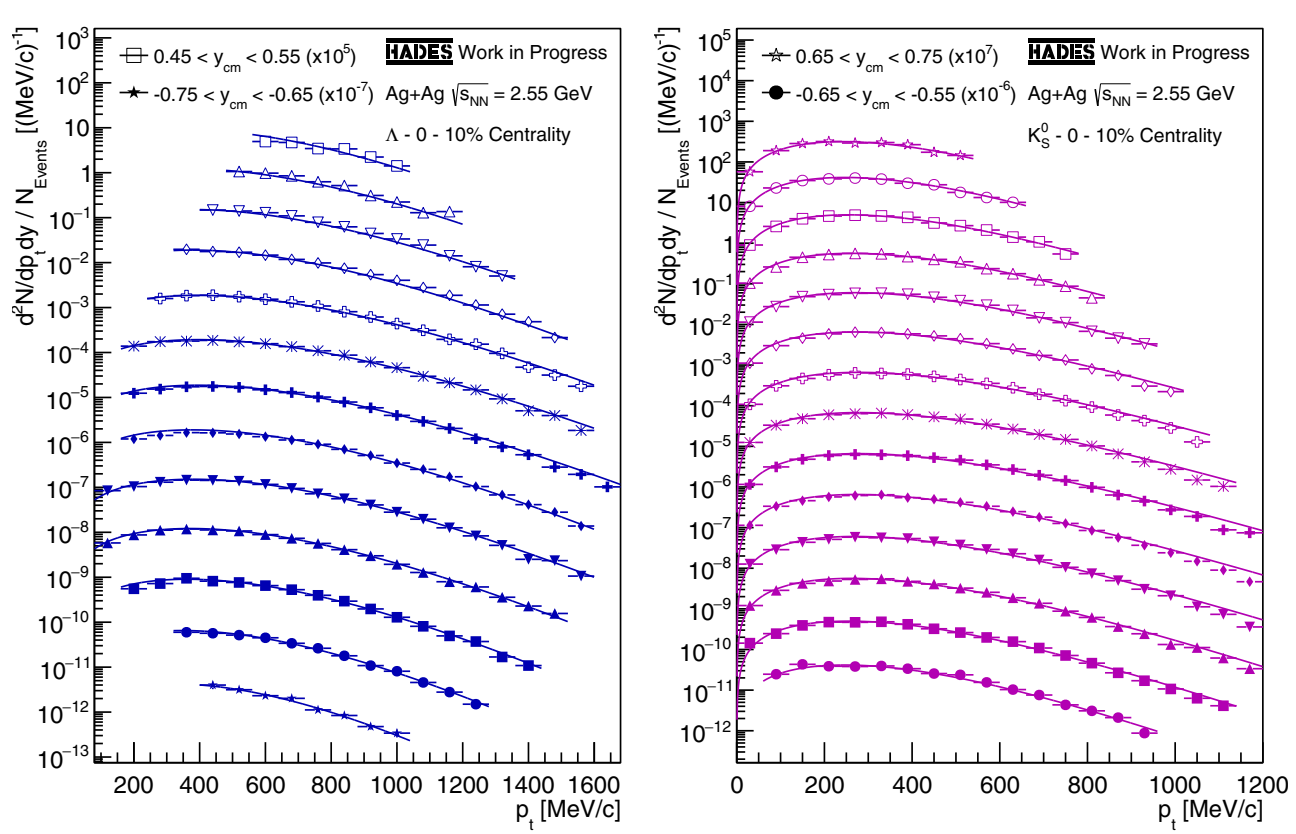

Figure 3. Transverse momentum spectra of $\Lambda$ hyperons (left) and $K_{S}^{0}$ mesons (right) from the $0-10 \%$ most central $\mathrm{Ag}(1.58 \mathrm{~A} \mathrm{GeV})+\mathrm{Ag}$ events measured by HADES.

\section{Multi-Differential Analysis}

The production of strange hadrons is analyzed multi-differentially in transverse momentum, rapidity and centrality. Figure 3 shows on the left side the transverse momentum spectra of $\Lambda$ hyperons in 13 rapidity intervals and on the right side the transverse momentum spectra of $K_{S}^{0}$ mesons in 14 rapidity intervals both for the $0-10 \%$ most central $\mathrm{Ag}(1.58 \mathrm{~A} \mathrm{GeV})+\mathrm{Ag}$ collisions. Thanks to the excellent acceptance of the HADES detector, both analyses cover a large proportion of the forward and backward hemisphere of the total phase-space which allows for in-depth studies of the production mechanisms of strangeness at SIS18 energies.

The measured transverse spectra are extrapolated to the regions of the total phase-space not covered by the analysis assuming the particles to be produced with Boltzmann distributed momenta. The corresponding fit functions, that are also depicted in figure 3 , describe the measured data over the entire range despite the simplicity of the underlying assumption.

Furthermore, the global polarization is measured using $\Lambda$ hyperons as probes. Likewise, this measurement is performed multi-differentially in transverse momentum, rapidity and centrality. In both heavy-ion collision systems measured by HADES, $\mathrm{Au}(1.23 \mathrm{~A} \mathrm{GeV})+\mathrm{Au}$ and $\mathrm{Ag}(1.58 \mathrm{~A} \mathrm{GeV})+\mathrm{Ag}$, a significant global polarization was measured unlike at high collision energies where the global polarization vanishes. The HADES global polarization measurement is described in more detail in the contribution [23] of this conference.

In addition to the $\Lambda$ hyperons and $K_{S}^{0}$ mesons, also the ${ }_{\Lambda}^{3} H$ was successfully reconstructed by HADES via its weak decay into a ${ }^{3} \mathrm{He}$ and a $\pi^{-}$in $\mathrm{Ag}(1.58 \mathrm{~A} \mathrm{GeV})+\mathrm{Ag}$ collisions. This is the first time the ${ }_{\Lambda}^{3} \mathrm{H}$ is observed at mid-rapidity at such low collision energies. As a hypernucleus, the ${ }_{\Lambda}^{3} \mathrm{H}$ is sensitive to the medium due to both production of strangeness in collective processes and the formation of nuclei from the baryon dominated matter. Furthermore, the 


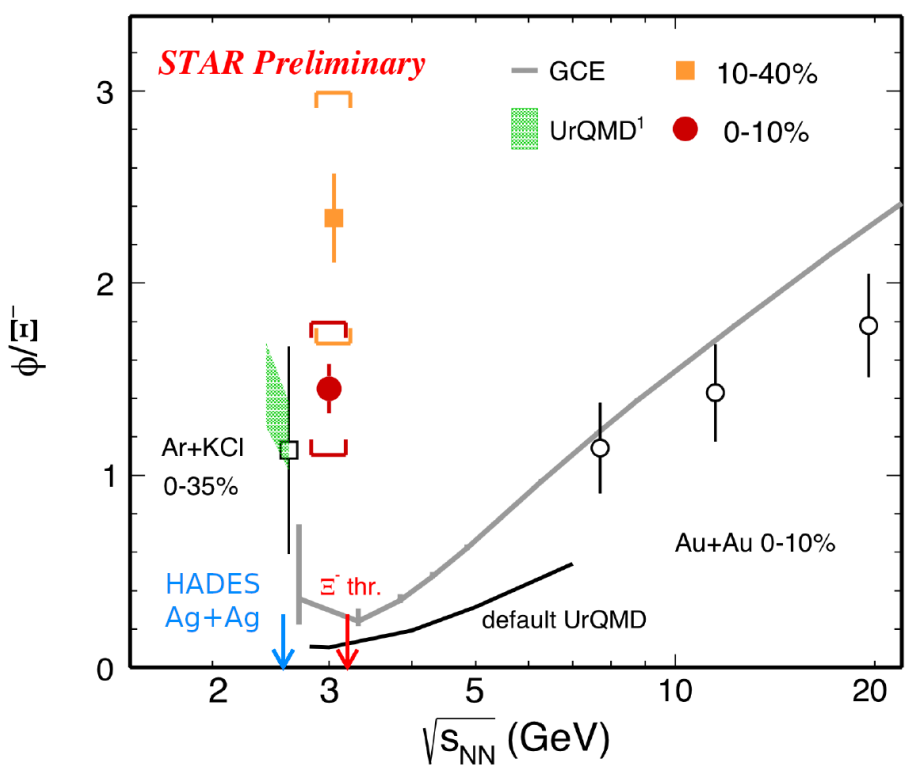

Figure 4. $\phi / \Xi^{-}$ratio in dependence of $\sqrt{\mathrm{s}_{\mathrm{NN}}}$ as presented by the STAR collaboration on this conference [14]. The red arrow on the $\mathrm{x}$-axis depicts the free nucleon nucleon threshold energy of $\Xi^{-}$ production and the blue arrow depicts the $\sqrt{\mathrm{s}_{\mathrm{NN}}}$ of the $\mathrm{Ag}(1.58 \mathrm{~A} \mathrm{GeV})+\mathrm{Ag}$ collisions measured by HADES. This emphasizes the need for more data in this energy regime.

study of hypernuclei allows to gain more information on hyperon nucleon interaction which are crucial to understand the influence of hyperons on the nuclear equation of state.

\section{Summary and Outlook}

In this contribution we presented first data from the HADES $\mathrm{Ag}(1.58 \mathrm{~A} \mathrm{GeV})+\mathrm{Ag}$ measurement campaign conducted as a part of the FAIR phase- 0 physics program. The combination of the high amount of recorded events and the sophisticated analysis methods allows for indepth studies of the production of (rare) strange hadrons. Furthermore, the ${ }_{\Lambda}^{3} H$ was measured for the first time at midrapidity at such low collision energies. In combination with the STAR fixed-target program in which $\mathrm{Au}+\mathrm{Au}$ collisions down to $\sqrt{\mathrm{S}_{\mathrm{NN}}}=3 \mathrm{GeV}$ were measured, the high baryon density region of the phase-diagram of QCD matter is explored in more detail than ever before.

Figure 4, which was presented by the STAR collaboration on this conference [14], shows the $\phi / \Xi^{-}$ratio in dependence of $\sqrt{\mathrm{S}_{\mathrm{NN}}}$. The plot shows results from the HADES $\operatorname{Ar}(1.76 \mathrm{~A} \mathrm{GeV})+\mathrm{KCl}$ measurement campaign and the STAR Au+Au fixed target program as well as theoretical predictions. In the HADES $\mathrm{Au}(1.23 \mathrm{~A} \mathrm{GeV})+\mathrm{Au}$ measurement campaign the $\phi$ was successfully measured [13], however, the $\Xi^{-}$could not be reconstructed successfully. In the HADES $\mathrm{Ag}(1.58 \mathrm{~A} \mathrm{GeV})+\mathrm{Ag}$ measurement campaign, thanks to the increased amount of recorded events, the increased energy and the decreased amount of background nucleons, we expect to reconstruct and analyze the $\phi$ mesons and the $\Xi^{-}$hyperons at lower energies than ever before.

In parallel to the ongoing analysis of the $\mathrm{Ag}(1.58 \mathrm{~A} \mathrm{GeV})+\mathrm{Ag}$ data, the HADES collaboration is implementing the Kalman-Filter based KF-Particle-Finder [24] package to reconstruct decaying particles. This approach is already used by STAR, ALICE and the future CBM experiment to increase the reconstruction efficiencies of decaying particles. Thereby, it will strongly enhance the reconstruction and analysis of strange hadrons, in particular the double strange $\Xi$ hyperons. 
SIP JUC Cracow, Cracow (Poland), 2017/26/M/ST2/00600; TU Darmstadt, Darmstadt (Germany), VHNG-823, DFG GRK 2128, DFG CRC-TR 211, BMBF:05P18RDFC1; Goethe-University, Frankfurt (Germany) and TU Darmstadt, Darmstadt (Germany), ExtreMe Matter Institute EMMI at GSI Darmstadt; NRNU MEPhI Moscow, Moscow (Russia), RFBR funding within the research project no. 1802-00086, Fundamental properties of elementary particles and cosmology No 0723-2020-0041; JLU Giessen, Giessen (Germany), BMBF:05P12RGGHM; IPN Orsay, Orsay Cedex (France), CNRS/IN2P3; NPI CAS, Rez, Rez (Czech Republic), MSMT LTT17003, MSMT LM2018112, MSMT OP VVV CZ.02.1.01/0.0/0.0/18_046/0016066;

\section{References}

[1] J. Adamczewski-Musch et al. HADES Collaboration, Nature Phys. 15 1040-1045 (2019).

[2] M. Hanauske, J. Steinheimer, L. Bovard, A. Mukherjee, S. Schramm et al., J. Phys. Conf. Ser. 878012031 (2017).

[3] C. Ecker, M. Järvinen, G. Nijs and W. van der Schee, Phys. Rev. D 101103006 (2020).

[4] V. Steinberg, J. Staudenmaier, D. Oliinychenko, F. Li, Ö. Erkiner and H. Elfner, Phys. Rev. C 99, 064908 (2019).

[5] J. Adamczewski-Musch et al. HADES Collaboration, Phys. Lett. B 793 457-463 (2019).

[6] D.B. Kaplan and A.E. Nelson, Phys. Lett. B 175 57-63 (1986).

[7] C.B. Dover and G.E. Walker, Phys. Rept. 89 1-177 (1982).

[8] C.H. Lee, G.E. Brown, D.P. Min and M. Rho, Nucl. Phys. A 585 401-449 (1995).

[9] F. Laue et al. KaoS Collaboration, Phys. Rev. Lett. 821640 (1999).

[10] M. Menzel et al. KaoS Collaboration, Phys. Lett. B 49526 (2000).

[11] A. Förster et al. KaoS Collaboration, Phys. Rev. Lett. 91152301 (2003).

[12] A. Forster, F. Uhlig, I. Bottcher, D. Brill et al., Phys. Rev. C 75024906 (2007).

[13] J. Adamczewski-Musch et al. HADES Collaboration, Phys. Lett. B 778 403-407 (2018).

[14] G. Xie et al. STAR Collaboration, SQM2021 conference talk (2021).

[15] G. Agakishiev et al. HADES Collaboration, Eur. Phys. J. A 5081 (2014).

[16] J. Adamczewski-Musch et al. HADES Collaboration, Phys.Rev.C 95015207 (2017).

[17] J. Adamczewski-Musch et al. HADES Collaboration, Phys. Rev. C 102024001 (2020).

[18] G. Agakishiev et al. HADES Collaboration, Eur. Phys. J. A 41 243-277 (2009).

[19] J. Podolanski and R. Armenteros, Phil. Mag. 45 13-30 (1954).

[20] A. Hocker, P. Speckmayer, J. Stelzer, J. Therhaag, E. von Toerne et al., TMVA - Toolkit for Multivariate Data Analysis, CERN-OPEN-2007-007 (2007).

[21] S. Spies, Master Thesis Goethe Universität Frankfurt (2018).

[22] S. Spies, J. Phys. Conf. Ser. 1667012041 (2020).

[23] F. Kornas et al. HADES Collaboration, these proceedings.

[24] S. Gorbunov and I. Kisel, CBM-SOFT-note-2007-003 (2007). 